\title{
Research And Realization of Aluminum Plate Surface Defects Classification Based on a Combination of BP Neural Network and SVM
}

\author{
Di Liu \\ School of Electrical Engineering and Automation QiLu \\ University of Technology \\ Jinan ,Shandong \\ sdili_liudi@163.com
}

\author{
Qinghua Li \\ School of Electrical Engineering and Automation QiLu \\ University of Technology \\ Jinan ,Shandong \\ lqh@qllu.edu.cn
}

\begin{abstract}
Based on a combination of BP neural network and SVM, aluminum plate surface defects classification was discussed. In order to detect the defects, the target image is binaried by adaptive threshold method. After binarizing the target image, extract the characteristic value of six kinds of aluminum plate surface defect images and formed twentyfour dimensional feature vector. The principle and algorithm of $B P$ neural network and support vector machine are introduced, given a way about combination of BP neural network and SVM, and about the important parameters optimization was carried out. The results verify the efficiency, accuracy and robustness of the algorithm about the BP neural network and support vector machine (SVM) classification of combining.
\end{abstract}

Keywords-aluminum surface defects; BP neural network; SVM; classification; combine

\section{INTRODUCTION}

China has become the world's aluminum industry, especially the major powers of the aluminum industry, with the domestic enterprises to high-end aluminum products, attention and development. Especially as aerospace, automobile, ship, weapons and other high-end products of raw materials, the quality of importance is selfevident[1]. However, in the aluminum production process, due to equipment wear and production of raw materials or the manufacturing process and other reasons, aluminum, with the surface will have some flaws, Such as: bubble, oil spots, scratches, holes, etc. Sheet for key parts of the airplane, car or other high-end electronic products, these defects is an important factor affecting the quality of products. It is necessary to strengthen the aluminum surface defect detection classification, to eliminate waste, reduce waste of raw materials, improve production efficiency.

The essence of classification of the aluminum plate surface defects is pattern recognition. The traditional pattern recognition method has low classification efficiency, easily missed, labor-intensive, real-time detection of defect types poor and small to effectively assess the surface quality of the product. Artificial neural networks and support vector classification method proposed to some extent alleviate the problem, but a single artificial neural network method is suitable for less categories, on multiple category overall recognition accuracy is not high, this algorithm is time-consuming, poor generalization ability and need more training samples. Support vector machine for multi-category classification, overall higher resolution, but for individual classes defect recognition rate is not very high, especially for oil spots and other complex class defects. So to improve the classification rate of oil spot defect recognition and overall identification classification rate has become an urgent problem.

To improve the classification rate of oil spot defect recognition and overall recognition classification rate can be improved on the hardware, aluminum plate surface defect detection system uses ultra-high performance CCD camera, and to address the impact of high reflective for aluminum surface defects correctly classified by changing hardware facilities. To some extent, this scheme can improve the classification rate of single aluminum plate surface defect recognition and overall recognition classification rate, but increases the hardware complexity and increase costs, it is not conducive to the needs of the aluminum surface defect detection classification system for small and medium enterprises to promote cold-rolled aluminum production use.

To solve the above problem, a way of combination of BP neural network and SVM has been proposed. Firstly, we detect the defects of target image and binarizing. Then Combine with the target image and the binary image to extract feature. According to extract feature vector, using the training set to train classification model. Finally, the trained classification models for classifying the test set. Fig .1 is a specific classification model diagram.

Geometric features, shape features and gray level these three kinds of feature are extracted as the aluminum plate surface defect information. Before feature extraction, and the need for defect binary image processing. Due to the reflections is relatively strong on the surface of the aluminum plate, this caused images of the pixel distribution is very uneven .Then, the better effect of binarization is difficult to achieve through passing one or several fixed threshold . Considering this kind of situation, it is need to be done according to the gradient threshold, so we use the adaptive threshold method for image segmentation. Adaptive thresholding make each pixel value of the defective area to 0 . The pixel value outside of 
target area is set to 255. The next step is combining the defect image and the binary image to extract features.

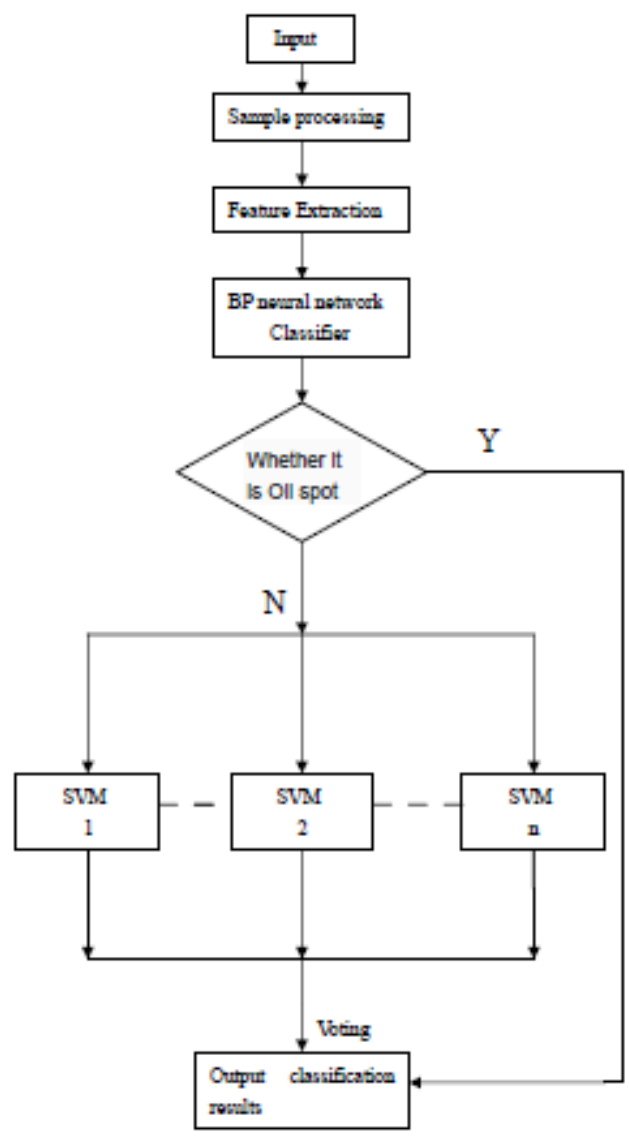

Figure 1. Defect classification model diagram

\section{FEATURE EXTRACTION}

In this paper, six kinds of defects on aluminum surface were classified. Six kinds of defects are shown in Fig .2. As follows: bubbles, damaged, oil spots, scratches, hole, black line.

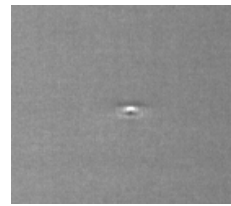

(a) bubble

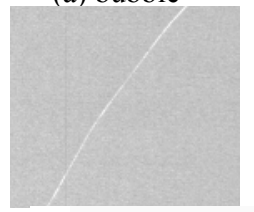

(d) scratch

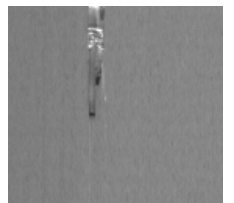

(b)damaged

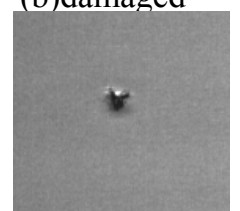

(e) holes

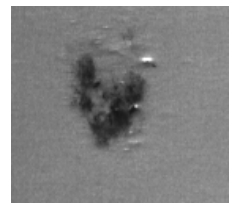

(c) oil spots

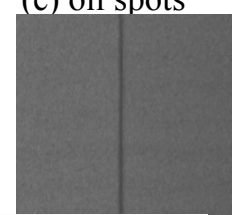

(f) black line
Figure 2. Six kinds of aluminum surface defects

Feature extraction is a key problem in aluminum plate surface defect classification. A valid set of feature vectors is an important guarantee for classification accuracy. Defect features are generally divided into geometry, shape feature, gray feature, where each category includes a variety of features. If you use all the features for training classification model and testing, not only have large computation, but also the final classification result is not the best, so it is necessary to extract some effective characteristic value. We extract 24-dimensional feature from the geometry, shape characteristics, gray features. This 24-dimensional feature as follows: defect area, defect aspect ratio, roundness, tightness, a seven order-order invariant moment to moment invariants[2], Fourier descriptors a Fourier descriptors to seven crooked degree, kurtosis, average gray value, variance, energy, fuzzy entropy.

\section{RECOGNITION OF DEFECT}

\section{A. The principle and algorithm of BP neural network}

BP (back propagation) network is a back propagation algorithm by former Multilayer feed-forward network, is currently the most widely used one neural network model[3]. The BP neural network algorithm is also known as the error back propagation algorithm. The algorithm process is composed of positive propagation and back propagation process. Forward propagation characteristic vector is based on the input learning samples, from the input layer, hidden layer and output layer of successive calculate the output of each node; Back propagation process is according to the results of the actual output of the output layer nodes and the ideal output error, in order to adjust the output layer, hidden layer and input layer weights between layers, until the error value to what we can accept within a threshold and convergence[4][5].

\section{B. The principle and algorithm of SVM [6][7]}

Support vector machine is a structural risk minimization principle of machine learning methods. Support vector machine (SVM) is the first in the case of linear separable for two categories of classification, and from the optimal classification theory. Its main principle is to find the optimal classification plane and maximize the classification interval between two classes of samples. Assumed in the two-dimensional space linear can be divided into two types of data, support vector machine (SVM) classification function is separated the two kinds of data as much as possible. In a sample set, support vector used to guide the optimization process, its number of sample input vector dimension there is no direct link, which can effectively avoid the curse of dimensionality because the sample data input feature vector dimension caused by excessive. For linear inseparable problem, kernel function of support vector machine (SVM) can map sample to a high-dimensional feature space, make its linear separable. General nuclear function mainly has polynomial kernel function, gaussian radial basis kernel function, the model S kernel function, etc. This article selects gaussian radial basis function as the kernel function, it can be represented as:

$$
k\left(x_{i}, x_{j}\right)=\exp \left(-\gamma\left\|x_{i}-x_{j}\right\|^{2}\right), \gamma>0
$$

Initially, Support vector machine proposed to solve binary classification problem, but in practice it often be sub category more than two. To solve the problem, the main idea is combination of several two classifiers to implement a multiple classifier. 


\section{The combination of BP neural network and support vector machine (SVM) classification model}

The establishment of the combined classification model, in order to improve the recognition rate of oil spots and overall defects. First, the rest of the oil spot outside defects (bubbles, damaged, scratches, holes, black line) as the first class defect. With oil spot and the first kind of defect samples for training the BP neural network model; Use of a bubble, damaged, scratches, holes, black line defect which five samples for training SVM model. The specific steps are as follows:

1) The selected training sample set for characteristic values of the normalized. For BP neural network, this article select oil spot defect sample figure of one hundred and twenty, the first kind of defect sample figure of one hundred and forty. In view of the support vector machine training sample set as shown in TABLE I.

TABLE I. SVM TRAINING SAMPLE SET

\begin{tabular}{|l|l|l|l|l|l|}
\hline Defect & Bubble & Damaged & Holes & Scratch & Black \\
\hline $\begin{array}{l}\text { Training } \\
\text { Set }\end{array}$ & 90 & 100 & 90 & 100 & 110 \\
\hline
\end{tabular}

24-dimensional feature value for each sample extraction, the characteristic value of the extracted range is very larges, some negative characteristic values reach dozens of square magnitude, in order to avoid a set of characteristic value of the maximum and minimum influence on classification model, each characteristic value normalization between $[1,1]$, the characteristic value of the normalized data as learning samples.

2) Select the appropriate structure and parameters, use of the training set to get BP neural network and SVM model. Take oil spot characteristic value and characteristic value of the first kind of defects as input of BP neural network model, So the structure of the BP neural network classification model chosen as three layers, including the input layer, hidden layer and output layer, the number of input layer nodes and the extraction of defect feature value dimension, same as twenty-four; Output layer node number is 2 , the number of hidden layer nodes is $6 \sim 15$. Build support vector machine model is to think of the BP neural network model is first class subdivisions defects, which is divided into bubbles, damaged, dark marks, light marks, holes, black line, these defects are classified using one of the methods, need to build 10 SVM model, the $\mathrm{n}$ in Fig. 1 is 10 .

3) Get classification model, the first classification using the trained BP neural network model to classify the test set, think of BP neural network as the defect of oil spot output classification as a result, the recognition is the first kind of defect is classified by the support vector machine (SVM) classification model. Using OpenCV for programming, remove samples which the BP neural network model considered the oil patch flaws, allow the BP neural network model is considered to be the first test set of defect into the support vector machine (SVM) classification model, the first class defects subdivided into bubbles, damaged, scratches, holes, black line by support vector machine, integrated BP neural network and support vector machine (SVM) classification results and output..

\section{The choice of kernel function and parameters optimization}

BP neural network's activation function is used in: threshold function, linear function, $\mathrm{S}$ function, radiation function, etc. BP network propagation algorithm is used to study the right values, using the gradient descent algorithm to minimize the network square error between actual output and target output as the goal [8]. Need to learn function differentiable, so choose $\mathrm{S}$ function in the hyperbolic tangent function as activation function. S function is:

$$
f(x)=\frac{1-e^{-x}}{1+e^{-x}}
$$

Other parameters of $\mathrm{BP}$ neural network are set as shown in TABLE II.

TABLE II. BP NEURAL NETWORK PARAMETER SETTINGS

\begin{tabular}{|c|l|l|l|}
\hline $\begin{array}{l}\text { Input layer } \\
\text { node number }\end{array}$ & $\begin{array}{l}\text { Number of } \\
\text { hidden layer }\end{array}$ & $\begin{array}{l}\text { Number of } \\
\text { hidden layer } \\
\text { nodes }\end{array}$ & $\begin{array}{l}\text { Output layer } \\
\text { node number }\end{array}$ \\
\hline 24 & 1 & $\mathrm{~N}$ & 2 \\
\hline $\begin{array}{l}\text { The largest } \\
\text { number of } \\
\text { iterations }\end{array}$ & $\begin{array}{l}\text { Error } \\
\text { precision }\end{array}$ & $\begin{array}{l}\text { Learning } \\
\text { Rate }\end{array}$ & $\begin{array}{l}\text { Momentum } \\
\text { factor }\end{array}$ \\
\hline 250000 & 0.00001 & 0.15 & 0.1 \\
\hline
\end{tabular}

Selection of the optimal number of hidden layer nodes, use the method - trial and error method [9] and combined with (3) to be set.

$$
m=\sqrt{n+l}+\alpha
$$

Where, $\mathrm{m}$ is hidden nodes; $\mathrm{n}$ is the input layer nodes; output nodes; a number between $1 \sim 10$. When $\alpha$ is different the overall defect recognition rate as shown in TABLE III.

TABLE III. WHEN DIFFERENT VALUES OF A OVERALL DEFECT RECOGNITION RATE

\begin{tabular}{|l|l|l|l|l|l|}
\hline$\alpha$ & 1 & 2 & 3 & 4 & 5 \\
\hline $\begin{array}{l}\text { Overall } \\
\text { recognition } \\
\text { rate }\end{array}$ & $91 \%$ & $89 \%$ & $92 \%$ & $91 \%$ & $94 \%$ \\
\hline$\alpha$ & 6 & 7 & 8 & 9 & 10 \\
\hline $\begin{array}{l}\text { Overall } \\
\text { recognition } \\
\text { rate }\end{array}$ & $93 \%$ & $89 \%$ & $90 \%$ & $96 \%$ & $89 \%$ \\
\hline
\end{tabular}

As can be seen from the table, $\alpha$ take 9 has the highest rate of defect recognition, so we set the hidden layer nodes are 14.

In this paper, support vector classification model adopting the way of subdividing the first kind of defect, we need to build ten sub-classification. And select radial basis function as kernel function of support vector machine. The kernel function is need to set the punishment factor $C_{\text {and }}$ parameter $\gamma$. For these two parameters setting, we apply LIBSVM package [10] by Taiwan university professor Lin designed and gnuplot software for optimization. The figure is to distinguish between bubbles and scratches 


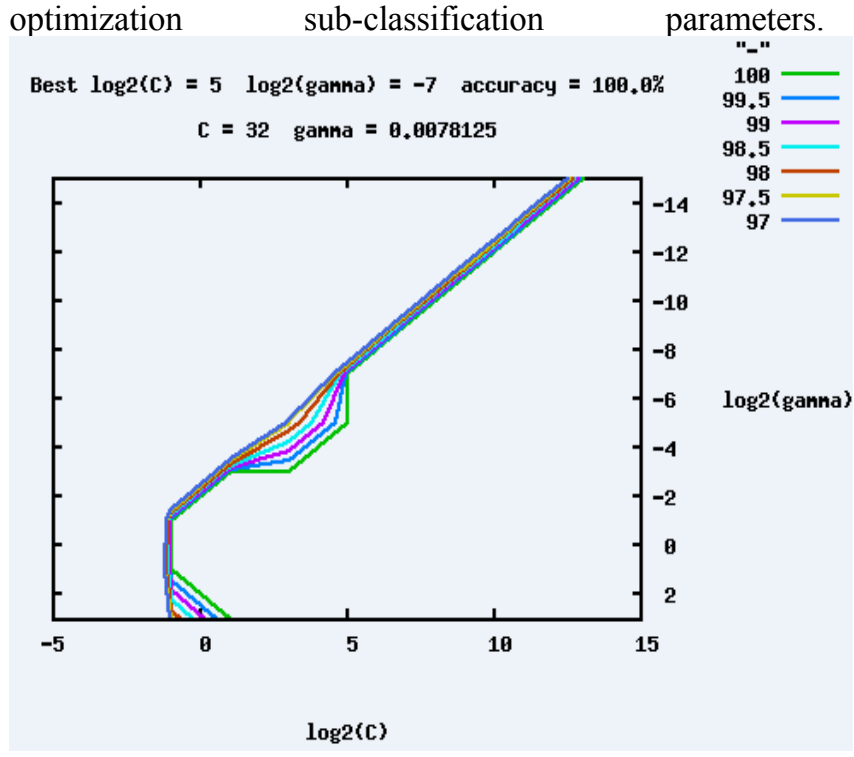

Figure 3. Distinguish between bubbles and scratches classifier parameters optimization

As can be seen from the figure above, the penalty factor and the optimal parameter values of the subclassifiers were: 32 and 0.0078125 . Optimal parameter values of the other sub-categories is also obtained with the same method and given in the following table.

TABLE IV. SUB-OPTIMAL PARAMETERS OF THE CLASSIFIER STATISTICS

\begin{tabular}{|l|c|c|l|}
\hline Sub-classifiers & $C$ & $\gamma$ & accuracy \\
\hline Bubble\&Damaged & 32768 & 0.03125 & $93.18 \%$ \\
\hline Bubble \& Black & 30 & 0.006273 & $94.24 \%$ \\
\hline Bubble \& Holes & 32 & 2 & $96.77 \%$ \\
\hline Damage\&Scratch & 2 & 0.5 & $100 \%$ \\
\hline Damage\& Black & 4 & 0.8 & $97.45 \%$ \\
\hline Damage \& Hole & 2048 & 0.03125 & $92.59 \%$ \\
\hline Scratch \& Black & 28 & 1.5 & $97.76 \%$ \\
\hline Scratch \& Holes & 2 & 0.5 & $100 \%$ \\
\hline Black \& Holes & 3 & 0.7 & $95.50 \%$ \\
\hline
\end{tabular}

\section{CLASSIFICATION RESULTS AND ANALYSIS}

Take six kinds of typical aluminum plate surface defect images of two hundred as the test set, the test result as shown in TABLE $\mathrm{V}$.
TABLE V. ClLASSIFICATION RESULTS

\begin{tabular}{|l|l|l|l|l|l|l|}
\hline Defect & $\begin{array}{l}\text { Oil } \\
\text { Spot }\end{array}$ & Bubble & Scratch & Damaged & Black & Hole \\
\hline $\begin{array}{l}\text { The test } \\
\text { sample }\end{array}$ & 40 & 30 & 34 & 32 & 30 & 34 \\
\hline $\begin{array}{l}\text { Recognition } \\
\text { rate }\end{array}$ & 38 & 30 & 33 & 31 & 28 & 34 \\
\cline { 2 - 7 } & $95 \%$ & $100 \%$ & $97.05 \%$ & $96.87 \%$ & $93.33 \%$ & $100 \%$ \\
\hline $\begin{array}{l}\text { The overall } \\
\text { recognition } \\
\text { rate }\end{array}$ & \multicolumn{7}{|c|}{$97 \%$} \\
\hline
\end{tabular}

As can be seen from TABLEV, using combination of BP neural network and support vector machine (SVM) classification method make the sizes, the classification of the irregular shape of oil spot defect recognition rate and defect recognition rate have reached a higher level. The combination of BP neural network and support vector machine (SVM) classification method to make full use of the BP neural network classifier performance is particularly good characteristics in oil stain classification results and support vector machine (SVM) to other classes defects have higher classification accuracy and good generalization performance. Combination of the two on the aluminium plate surface defect classification has played a good effect.

\section{ACKNOWLEDGMENT}

This work was supported by the Shandong Province Science and Technology Development Plan (No. 2013GGX10132), National Natural Science Foundation of China (No.41204025), the National Postdoctoral fund (No.2012M520967) and the Shandong Province Young Scientists Fund (No.BS2013DX031).

\section{REFERENCES}

[1] Yaqing Xiao, "Development of Chinese aluminum industry," Beijing: Metallurgical Industry,2007,pp.5-16.

[2] Qin zhong,"Vehicle clasification based on moment invariant feature and BP neural network,". Journal of South China University of Technology,vol(37),pp.87-91,2009.

[3] Nawi N M, Ransing R S, Sallen, M N M, et al, "An improved back propagation neural network agorithm on classification problems,".2010,pp.177-188.

[4] Davies E R. Machine Visio n, "Theory, Algorithms, Practicalities," Beijing : Posts \& Telecom ,2009.

[5] Xulei, Liujian, "Classifying remote sening image based on BP neural network technology,"Journal of Geospatial Information Science,vol(10),pp.83-85,2012

[6] Huijun $\mathrm{Hu}$, yuanxiang Li, Maofu Liu, and Wenhao Liang, "The strip surface defects classification method based on machine learning research," . Computer engineering and design, 2014, pp . 620-624.

[7] Ronny L, Alexandred, "Support vector machine classification with indefinite kernels," Mathematical Programming Computation , 2009 ,pp.97-118.

[8] Zhang zheng,Yanping Wang,Guixiang Xue, "Digital image processing and machine vision ," Beijing: people's posts and telecommunications , 2010,pp.450-453.

[9] Liqun Han, "Artificial neural network theory, the design and application," .Beijing: chemical industry , 2007,pp.55-62

[10] Chang C C, Lin C J, "LIBSVM: A library for support vector machines ,"ACM Transactions on intelligent Systems and Technology, vol.27,pp.21-27, 2011 